\title{
El triaje en los servicios de urgencias en hospitales de la subregión suroeste de Antioquia. Estudio observacional descriptivo
}

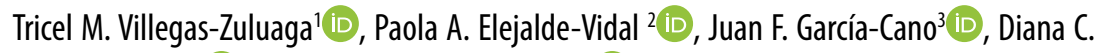
Martínez-Pérez ${ }^{4}$ iD, Carlos E. Vallejo-Bocanumen ${ }^{5}$ (D)

\footnotetext{
${ }^{1}$ Especialista en Medicina de Urgencias, IPS Universitaria - Clínica León XIII. Grupo de Investigación en urgencias y emergencias (GIURE), Universidad de Antioquia.

2 Tecnóloga en Atención Prehospitalaria, Especialista en sistemas de preparativos para Emergencias y Desastres. Docente, Facultad de Medicina, Universidad de Antioquia.

${ }^{3}$ Administrador de servicios de salud. Grupo de Investigación en urgencias y emergencias (GIURE). Estudiante Maestría en Salud Pública, Universidad de Antioquia.

${ }^{4}$ Médica y Cirujana. Magíster en Epidemiología Clínica. Grupo de Investigación en urgencias y emergencias (GIURE), Universidad de Antioquia.

${ }^{5}$ Coordinador Grupo de Investigación en urgencias y emergencias (GIURE), Universidad de Antioquia. Profesor asociado, Facultad de Medicina, Universidad de Antioquia. Especialista en Medicina de Urgencias, IPS Universitaria - Clínica León XIII.
}

\section{INFORMACIÓN DEL ARTÍCULO}

\section{Palabras clave}

Implementación de Plan de Salud;

Medicina de Emergencia;

Servicio de Urgencia en Hospital;

Triaje

Recibido: junio 9 de 2021

Aceptado: noviembre 24 de 2021

\section{Correspondencia:}

Carlos Eduardo Vallejo-Bocanumen; eduardo.vallejo@udea.edu.co

Como citar: Villegas-Zuluaga TM, ElejaldeVidal PA, García-Cano JF, Martínez-Pérez DC, Vallejo-Bocanumen CE. El triaje en los servicios de urgencias en hospitales de la subregión suroeste de Antioquia. Estudio observacional descriptivo. latreia [Internet]. 2023 Abr-Jun; 36:(2):158-168. https://doi.org/10.17533/udea. iatreia.162

\section{(c) $\$(0)$}

Copyright: (c) 2023

Universidad de Antioquia.

\section{RESUMEN}

Objetivo: el objetivo de este estudio fue identificar la implementación del triaje en los servicios de urgencias hospitalarios, posterior a la divulgación de la resolución 5596 de 2015 del Ministerio de Salud de Colombia, en los hospitales de la subregión Suroeste del departamento de Antioquia.

Métodos: estudio observacional descriptivo, tipo corte transversal. Se incluyeron los 23 hospitales públicos de los municipios de la subregión suroeste de Antioquia. Se utilizó muestreo no probabilístico, por conveniencia. Los datos se obtuvieron mediante encuesta y técnica de entrevista estructurada.

Resultados: la encuesta fue respondida por funcionarios de los hospitales de los 23 municipios del suroeste antioqueño. Sólo 3 instituciones (13\%) tienen una adecuada implementación de resolución 5596 (política de triaje) del ministerio de salud (adherencia a la resolución mayor al $80 \%$ ), el $74 \%$ reportaron una adherencia a la resolución $>20 \%$ y $\leq 80 \%$ y 3 instituciones no lograron un porcentaje de adherencia mayor al $20 \%$ a la resolución. Sólo 3 instituciones (13\%) utilizan triaje validado, en otras 3 instituciones (13\%) no saben cuál tipo de triaje usan y en la mayoría de las instituciones (74\%) emplean un triaje no validado internacionalmente.

Conclusiones: a pesar de ser una normativa de obligatorio cumplimiento por parte de todos los hospitales del territorio nacional, sólo el $13 \%$ de los hospitales incluidos en el estudio cumplen con los criterios técnicos de la política de triaje del Ministerio de Salud de Colombia. 


\section{Triage in emergency wards of the Southwest subregion of Antioquia. Descriptive observational study}

Tricel M. Villegas-Zuluaga' (D), Paola A. Elejalde-Vidal ${ }^{2}$ iD, Juan F. García-Cano3 ${ }^{(D)}$, Diana C. Martínez-Pérez ${ }^{4}$ iD, Carlos E. Vallejo-Bocanumen ${ }^{5}$ (D)

1 Specialist in Emergency Medicine, IPS Universitaria - Clínica León XIII. Urgency and Emergency Research Group (GIURE), University of Antioquia.

${ }^{2}$ Technician in Prehospital Care, Specialist in Preparation Systems for Emergencies and Disasters. Professor, Faculty of Medicine, University of Antioquia.

${ }^{3}$ Health services administrator. Urgency and Emergency Research Group (GIURE). Master's student in Public Health, University of Antioquia.

${ }^{4}$ Physician and Surgeon. Master in Clinical Epidemiology. Urgency and Emergency Research Group (GIURE), University of Antioquia.

${ }^{5}$ Coordinator of the Urgency and Emergency Research Group (GIURE), University of Antioquia. Associate Professor, Faculty of Medicine, University of Antioquia. Specialist in Emergency Medicine, IPS Universitaria - Clínica León XIII.

\section{ARTICLE INFORMATION}

\section{Keywords}

Emergency Medicine;

Health Plan Implementation;

Hospital Emergency Service;

Triage

Received: June 9, 2021

Accepted: November 24, 2021

\section{Correspondence:}

Carlos Eduardo Vallejo-Bocanumen; eduardo.vallejo@udea.edu.co

How to cite: Villegas-Zuluaga TM, ElejaldeVidal PA, García-Cano JF, Martínez-Pérez DC, Vallejo-Bocanumen CE. Triage in emergency wards of the Southwest subregion of Antioquia. Descriptive observational study. latreia [Internet]. 2023 Abr-Jun;36:(2):158-168. https://doi.org/10.17533/udea.iatreia.162

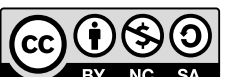

Copyright: (c) 2023

Universidad de Antioquia.

\section{ABSTRACT}

Objective: The objective of this study was to identify the implementation of triage in emergency wards, after the release of Resolution 5596 of 2015 of the Ministry of Health of Colombia, in the hospitals of the Southwest subregion of the department of Antioquia.

Methodology: Descriptive, cross-sectional study. The 23 public hospitals in the municipalities of the southwestern subregion of Antioquia were included. A convenience non-probabilistic sampling was used. The data were obtained through a survey and structured interview technique.

Findings: The survey was answered by hospital officials from the 23 municipalities of southwest Antioquia. Only 3 institutions (13\%) have an adequate implementation of the triage service (adherence to the resolution greater than $80 \%$ ), $74 \%$ reported adherence to the resolution $>20 \%$ and $\leq$ $80 \%$ and 3 institutions do not achieved a percentage of adherence greater than 20\% to the resolution. Only 3 institutions (13\%) use validated triage, in 3 other institutions (13\%) they do not know what type of triage they use and in most institutions (74\%) they use a non-validated triage.

Conclusions: in spite of being a mandatory regulation for the hospitals of the national territory, only $13 \%$ of the hospitals included in the study comply with the technical criteria of triage policy of the Colombian Ministry of Health. 


\section{Introducción}

El triaje, definido por la Organización Mundial de la Salud (OMS) como la clasificación de los pacientes en grupos prioritarios, de acuerdo a sus necesidades y a los recursos disponibles, es un proceso clave en la organización de la atención de los Servicios de Urgencias en Hospitales (SUH). Su objetivo principal es asegurar que el paciente reciba el nivel de prioridad acorde a las necesidades clínicas, para garantizar la seguridad, la optimización de los recursos y una atención en salud de calidad (1-3).

Desde hace más de 40 años distintos sistemas de salud del mundo han implementado el triaje en los SUH, como una herramienta para ordenar e iniciar la atención de los pacientes que solicitan asistencia médica en los SUH y con ello disminuir el riesgo de distinto desenlaces adversos, incluida la muerte (1,3-9). En Colombia no se cuentan con antecedentes claros acerca de la implementación del triaje, y solo hasta el año 2015 por medio del Sistema General de Seguridad Social en Salud y acorde con los lineamientos del Ministerio de Salud y Protección Social, mediante Resolución 5596 del 2015, se establece que todos los hospitales o Instituciones Prestadoras de Servicios de Salud (IPS), y las entidades responsables del pago de los servicios de salud, están obligadas a implementar y cumplir con un triaje en los SUH. Dicha norma, además, define criterios técnicos para la implementación del triaje hospitalario (10). Sobre la base de este marco normativo, se realizan esfuerzos por mejorar los servicios de salud, la prestación en los SUH y mejorar los indicadores de proceso y resultado en las IPS (11). Si bien en los hospitales de alta complejidad se vienen realizando esfuerzos en implementar y medir los efectos de la política de triaje del Ministerio de Salud (12-14), al día de hoy se desconoce el estado de implementación de la misma en los hospitales de mediana y baja complejidad.

Antioquia cuenta con 9 subdivisiones territoriales y una de ellas, la subregión Suroeste, evidencia indicadores preocupantes en materia de salud pública; con tasas de mortalidad superiores a las departamentales, por infarto agudo de miocardio, menores de 5 años con enfermedad diarreica aguda y relacionada con el embarazo, parto y puerperio. Esta subregión cuenta con 23 hospitales, 22 de baja complejidad y uno de mediana complejidad, y prestan los servicios de salud aproximadamente a 377.482 habitantes de este territorio $(15,16)$.

El objetivo de este estudio es identificar la implementación del triaje en los SUH, posterior a la divulgación de la Resolución 5596 de 2015 del Ministerio de Salud y Protección Social de Colombia, en los hospitales públicos de la subregión Suroeste del departamento de Antioquia en el 2018.

\section{Metodología}

El estudio realizado es de tipo observacional descriptivo, tipo corte transversal. Se incluyeron todos los hospitales públicos de los municipios de la subregión suroeste de Antioquia.

El instrumento de recolección de la información fue desarrollado por el grupo investigador. Se realizó un piloto en un hospital universitario de alta complejidad de la ciudad de Medellín, en el que se determinó la comprensión de las preguntas y de la terminología utilizada, lo cual permitió afinar la encuesta.

Se solicitó autorización a los directores o gerentes de los hospitales para la participación en el estudio de cada hospital. La información se recolectó mediante encuesta estructurada, la cual fue administrada por personal previamente entrenado en dicha tarea. Esta fue respondida por los funcionarios de cada hospital -personal médico o de enfermería- encargados del proceso de triaje en el SUH. Las encuestas fueron realizadas de manera presencial en los SUH de cada hospital. Todas las respuestas de los encuestados debían estar acompañadas con evidencia física o documental que las respaldaran. 
El periodo de estudio fue entre septiembre de 2018 y febrero 2019.

Para el control de calidad de los datos se realizó un proceso de codificación a las preguntas abiertas, verificación de la información registrada y doble tabulación de los datos. Se almacenó la información en archivo de Microsoft Excel.

\section{Análisis de la información}

Se realizó análisis descriptivo de los datos. Se identificaron los tipos de triaje en cada institución, el número de niveles de triaje empleados, registro del triaje en la historia clínica, designación de tiempos según nivel de triaje, capacitación del personal encargado del triaje, información al paciente y reclasificación de los pacientes. Se obtuvieron las frecuencias relativas por cada variable.

Para establecer una implementación completa de la resolución se deben cumplir 6 aspectos: número de niveles de la escala triaje empleado (igual a cinco), registro del triaje en historia clínica, la asignación de tiempos para la atención según nivel de clasificación, capacitación del personal en la realización del triaje, brindar información al paciente acerca del proceso de triaje y hacer reclasificación de los pacientes. El cumplimiento de cada uno de los ítems representa un 16,6 \% de la implementación completa.

Se indagó acerca de las características del personal que implementa el triaje, y las características técnicas y de sistematización de cada uno de los SUH.

Adicionalmente, se determinó la inspección, vigilancia y control realizada al proceso de triaje, verificando el número de auditorías realizadas por entes reguladores en el último año a los SUH. Se presentan los estadísticos descriptivos, según la naturaleza de la variable.

\section{Consideraciones éticas}

El proyecto fue aprobado mediante el acta 007 del 10 de mayo del 2018 por el Comité de Ética del Instituto de Investigaciones Médicas de la Universidad de Antioquia. Este estudio fue considerado de riesgo mínimo en tensiones éticas que vulnere los derechos y el bienestar de los participantes. Se solicitó a cada representante del hospital el diligenciamiento del consentimiento informado, previo a la participación en el estudio.

\section{Resultados}

Se aplicó la encuesta en los hospitales públicos (Empresas Sociales del Estado) de los 23 municipios de la subregión suroeste de Antioquia, que fue respondida por personal del área de salud, médico o auxiliar de enfermería, encargados del proceso de triaje en los SUH en 22 instituciones (96\%); y en una institución fue respondida por el gerente del hospital (4\%).

En la Tabla 1 se presentan los 6 criterios técnicos planteadas por la resolución 5596 de 2015 para la adecuada implementación del triaje. De estos, "Personal capacitado en triaje/certificado de asistencia"y"se realiza reclasificación a los pacientes" fueron las categorías de menor cumplimiento por los SUH. Y las categorías "Registro de triaje en historia clínica"y "Brindar información al usuario acerca del sistema de triaje" fueron las de mayor cumplimiento. Se identificó que en el 78,3 \% de los SUH el personal encargado del triaje es médico general y en el 21,7 \% auxiliares de enfermería. Del total de personal que realiza el triaje, el $78 \%$ cuenta con experiencia específica en el servicio de triaje mayor a seis meses, de los cuales sólo el 13 \% refirió tener entrenamiento certificado. Adicionalmente, se encontró que el 87 \% de los hospitales cuenta con historia clínica electrónica y el 61 \% dice emplear un triaje de 5 niveles. 
Tabla1. Criterios técnicos para la implementación del triaje estructurado de 5 niveles, según política de triaje del Ministerio de Salud de Colombia

\begin{tabular}{|c|c|c|c|c|c|}
\hline \multirow{2}{*}{$\begin{array}{l}\text { Criterios para } \\
\text { implementación } \\
\text { de triaje }\end{array}$} & \multicolumn{2}{|c|}{ Total, hospitales } & \multirow{2}{*}{$\begin{array}{c}\text { Implementación } \\
\quad \geq \mathbf{8 0} \%\end{array}$} & \multirow{2}{*}{$\begin{array}{l}\text { Implementación } \\
>20 \% 0<80 \%\end{array}$} & \multirow{2}{*}{$\begin{array}{c}\text { Implementación } \\
\leq 20 \%\end{array}$} \\
\hline & n: 23 & $\%$ & & & \\
\hline \multicolumn{6}{|c|}{ Número de niveles de la escala triaje } \\
\hline 5 niveles & 14 & 60,9 & 3 & 11 & 0 \\
\hline 4 niveles & 7 & 30,4 & 0 & 6 & 1 \\
\hline 3 niveles & 2 & 8,7 & 0 & 0 & 2 \\
\hline \multicolumn{6}{|c|}{ Registro de triaje en historia clínica } \\
\hline Sí & 19 & 82,6 & 3 & 16 & 0 \\
\hline No & 4 & 17,4 & 0 & 1 & 3 \\
\hline \multicolumn{6}{|c|}{ Se asignan tiempos para la atención } \\
\hline Sí & 13 & 56,5 & 3 & 10 & 0 \\
\hline No & 10 & 43,5 & 0 & 7 & 3 \\
\hline \multicolumn{6}{|c|}{ Personal capacitado en triaje/certificado de asistencia } \\
\hline Sí & 3 & 13,0 & 1 & 2 & 0 \\
\hline No & 20 & 87,0 & 2 & 15 & 3 \\
\hline \multicolumn{6}{|c|}{ Se brinda información al usuario acerca del sistema de triaje } \\
\hline Sí & 19 & 82,6 & 3 & 14 & 2 \\
\hline No & 4 & 17,4 & 0 & 3 & 1 \\
\hline \multicolumn{6}{|c|}{ Se realiza reclasificación a los pacientes } \\
\hline Sí & 5 & 21,7 & 3 & 2 & 0 \\
\hline No & 18 & 78,3 & 0 & 15 & 3 \\
\hline
\end{tabular}

Fuente: creación propia

Al indagar por el tipo de triaje empleado en los SUH (ver Figura 1), se identificó que sólo 3 (13\%) utilizan triaje internacionalmente validado (ESI -Índice de Severidad de Emergencia, por sus siglas en inglés, ATS -Puntaje de Triaje Australiano, por sus siglas en inglés-, o MTS -Puntaje de Triaje de Manchester, por sus siglas en inglés-), en otros 3 (13\%) no saben cuál tipo de triaje usan, y en el resto de SUH (74 \%) emplean un triaje no validado internacionalmente y hacen referencia al "triaje que define la ley", "la resolución 412", a "el del sistema de la historia clínica", entre otras, como el tipo de triaje implementado. 


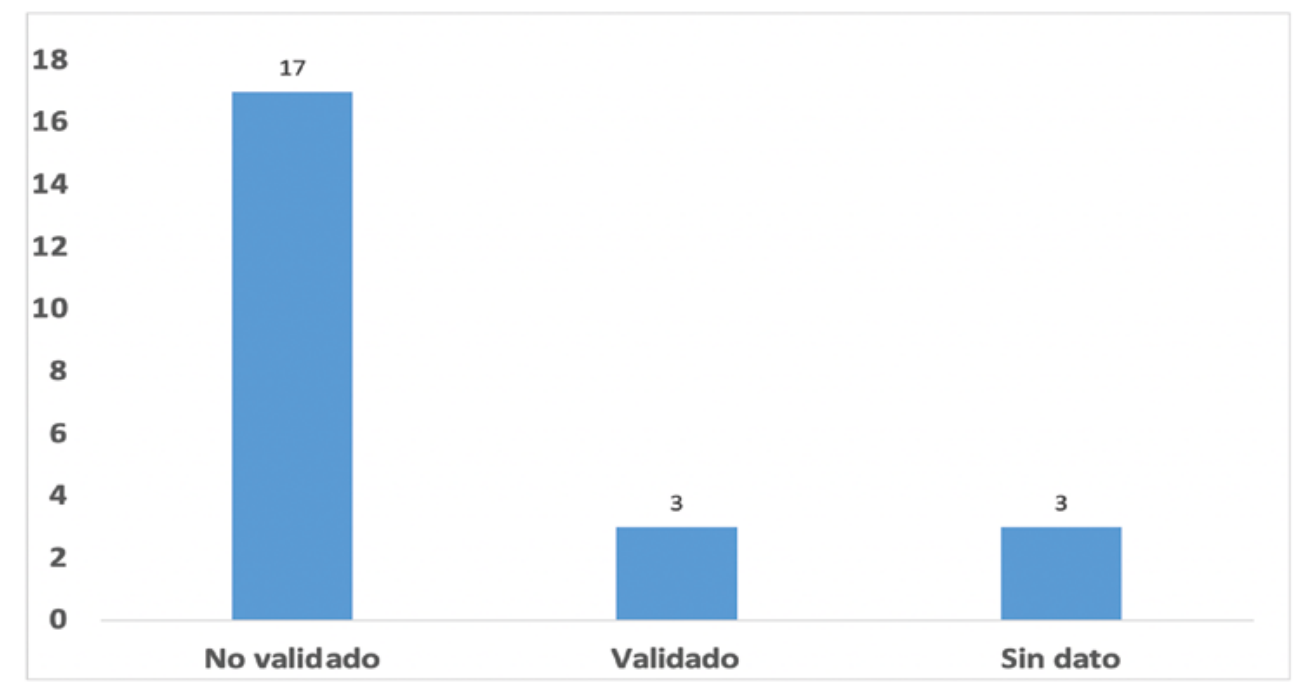

Figura 1. Distribución de los SUH del suroeste de Antioquia y el tipo de triaje utilizado.

Fuente: creación propia

En la Figura 2 se presentan los grupos SUH de acuerdo a la proporción implementación alcanzado, identificándose que sólo 3 (13\%) tienen una implementación mayor o igual al 80 \% del servicio de triaje (cinco o más criterios), el $74 \%$ reportaron una adherencia a la resolución > $20 \%$ y $\leq 80 \%$ (dos a cuatro criterios) y 3 de los SUH no lograron un porcentaje de adherencia mayor al $20 \%$ a la resolución (uno o ningún criterio).

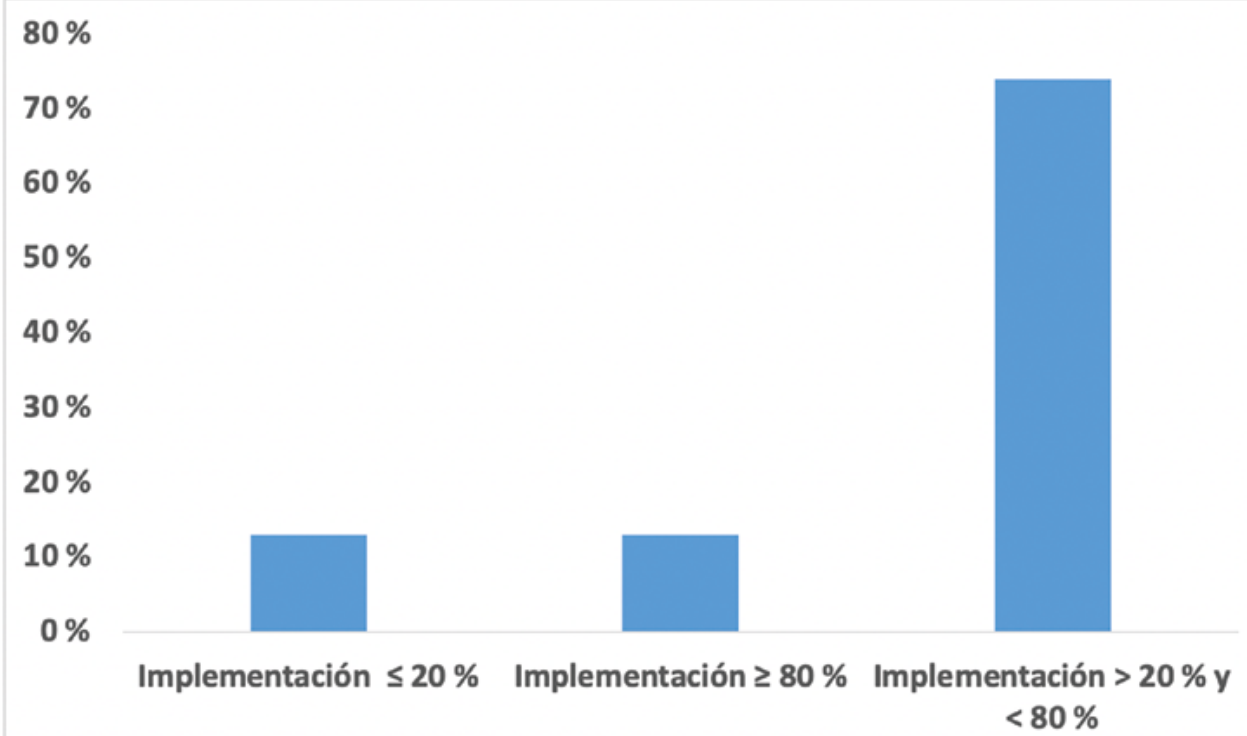

Figura 2. Distribución porcentual según implementación de la política de triaje de los hospitales del suroeste de Antioquia.

Fuente: creación propia 
Al indagar por la inspección, vigilancia y control que realizan a los SUH por parte de los entes de control -Secretaría de Salud Departamental y Municipal- se identifica que en 6 SUH (26 \%) se había realizado algún tipo de auditoría relacionada en el último año. Adicionalmente, se identificó que, aunque 12 hospitales (52,2\%) informaron el número de pacientes atendidos en el SUH durante el año 2017, ninguno tenía información del número total de triajes realizados en el mismo año.

\section{Discusión}

Este estudio evidencia que, aunque la política de triaje del Ministerio de Salud de Colombia es una norma de obligatorio cumplimiento para todas las clínicas y hospitales en el territorio nacional que cuenten con SU, sólo el 13 \% de las instituciones encuestadas cumplen, de manera adecuada, con los criterios técnicos propuestos por la resolución ministerial (10).

Se ha evidenciado que más de la mitad de los pacientes que acceden a los sistemas de salud y a cuidados al interior de los hospitales lo hacen a través de los SUH, generando con frecuencia fenómenos de sobresaturación $(3,17)$. La sobresaturación aumenta el riesgo de tiempos de espera prolongados para la atención, intervenciones inseguras, insatisfacción en pacientes y familiares e ineficiencia en el uso de los recursos (18-21); y aunque este fenómeno se encuentra lejos de ser resuelto, se han ideado e implementado estrategias para mitigar los riesgos mencionados, una de las cuales ha sido el triaje (2,22-24). Los objetivos principales del triaje son definir quien será atendido en primer lugar y el tiempo de espera para la atención de los pacientes, de acuerdo con la clasificación que suministra dicho proceso $(1,3)$. En este estudio se encontró que el 61 \% de los SUH dicen emplear un triaje de 5 niveles; sin embargo, el 74 \% emplea un triaje no validado internacionalmente, lo cual representa grandes retos y probables dificultades en términos de fiabilidad y estimación del riesgo, lo que podría significar errores en la clasificación real de la urgencia (25). Aunque la política de triaje del Ministerio de Salud no obliga a la implementación de una escala de triaje validada, debe tenerse presente que las escalas no validadas pueden subestimar o sobrestimar la clasificación de triaje, lo cual puede convertirse en una nueva barrera para la atención en salud con calidad y limitar el logro de los objetivos propuestos por la misma norma ministerial.

Gestionar una adecuada organización del cuidado al paciente en el SUH requiere, entre otros, de documentación adecuada de la atención y el registro real de los datos relacionados con dicho proceso. Los países de medianos y bajos ingresos enfrentan grandes retos para ello, lo cual se refleja en la escasa literatura relacionada con el tema en dichos países (26). La cuantificación de los tiempos de espera y la reclasificación de los pacientes son elementos importantes para el análisis y la gestión del cuidado en el SUH $(23,27)$. En este estudio evidenciamos que, si bien una proporción importante de los SUH encuestados respondieron tener tiempos estandarizados para la atención e informan a los pacientes de los tiempos de atención esperados para cada nivel de triaje, la medición de los tiempos de espera entre el triaje y la atención médica no se encuentran sistematizada, lo que podría generar subregistros sistemáticos y poca fiabilidad del dato, lo cual limita su uso para la gestión de los SUH. Adicionalmente, el 78 \% de los SUH no realiza en su triaje reclasificación de los pacientes que llevan más de una hora en espera. Así mismo, no se pudo documentar el número de pacientes que consultaban a triaje durante los periodos solicitados, la relación de los niveles de triaje asignada con el número de consultas en el SUH y el tiempo de atención promedio para cada nivel de triaje. A pesar de tener historia clínica electrónica, la mayoría de las instituciones no cuenta con información sistematizada que les permita obtener datos de calidad en forma oportuna para el análisis. El $26 \%$ (seis) de los SUH encuestados informaron haber tenido algún tipo de auditoría en el último año; sin embargo, al solicitar la documentación a los entes de vigilancia y control no se obtuvo respuesta. En este punto, la articulación entre Universidad, Empresa y Estado, a través tecnologías de la información y comunicación que faciliten el registro y la gestión del dato, permitiría 
mejorar la calidad de la información y optimizar los recursos, asegurando una adecuada y oportuna atención para los pacientes.

En este estudio se evidenció que en la mayoría de los SUH el personal sanitario que realiza el triaje es el mismo que realiza la atención médica, desarrollando dichas tareas de manera simultánea. Esta práctica puede considerarse insegura y peligrosa, ya que el dilatar el tiempo de espera para los pacientes que aguardan el triaje puede significar la prolongación de la oportunidad en la atención, que en enfermedades tiempo-sensibles está en estrecha relación con desenlaces adversos (28,29).

La implementación de protocolos de triaje adecuados garantiza que los pacientes que consultan a SUH reciban una atención con calidad. Se ha evidenciado que la implementación adecuada de la política del triaje del Ministerio de Salud y Protección Social de Colombia tiene efectos positivos sobre la calidad en la atención de los pacientes que acuden a los SUH (30). En clínicas y hospitales de baja complejidad hay dificultades para cumplir con protocolos de triaje, particularmente por limitación de recursos. En torno a esta barrera, la literatura científica plantea una serie de estrategias para tratar de mitigar su efecto, entre ellas la realización del triaje por un equipo y no por un único profesional en enfermería, pudiendo mejorar el flujo de pacientes y reducir la mortalidad en el SUH (31). El triaje debería realizarse por personal de la salud con experiencia y habilidades en pensamiento crítico y en este estudio evidenciamos que una considerable proporción del talento humano encargado de la realización del mismo no cumplen dicho perfil, dado con entrenamiento específico certificado en toma de decisiones y sistemas de clasificación de pacientes en los SUH (32). La profundización en el conocimiento relacionado con la medicina de urgencias también debe estar orientada a la formación de nuevos profesionales en medicina y enfermería con mayores y mejores capacidades, en aras de mejorar la seguridad, la experiencia de los pacientes y la optimización de recursos (33).

Por último, es indispensable asumir el triaje como un proceso inherente a la atención médica en el sistema de salud, que debe ser implementado de manera seria y evaluado bajo parámetros de calidad en la atención y el cuidado de nuestros pacientes $(34,35)$.

\section{Conclusiones}

Se evidenció que, a dos años de la divulgación de la resolución 5596 de 2015 del Ministerio de Salud de Colombia que establece los criterios técnicos para el triaje en los SU, hay una baja implementación de dicha política en los hospitales de la subregión suroeste del departamento de Antioquia.

Los hospitales deben aumentar sus esfuerzos en la implementación del triaje estructurado de cinco niveles, basados en los criterios técnicos de la política ministerial, con la finalidad de optimizar el acceso al sistema de salud y disminuir los riesgos asociados a los tiempos prolongados en la atención.

\section{Limitaciones}

Un potencial sesgo de información pudo derivarse del entrevistador; que intentó controlarse mediante el entrenamiento del personal encargado de administrar la encuesta y con el uso de un cuestionario estructurado. Igualmente, los encuestados pudieran intentar adecuar las respuestas para satisfacer la encuesta; sin embargo, cómo se mencionó en la metodología, los encuestadores debían solicitar la evidencia o la documentación que demostrara la respuesta. Los hallazgos de este estudio no son necesariamente representativos de los hospitales públicos de las restantes 8 subregiones del departamento de Antioquia. 


\section{Agradecimientos}

1. Comité para el Desarrollo de la Investigación - CODI, y Vicerrectoría de Investigación de la Universidad de Antioquia, por la financiación del proyecto de investigación, a través de la Convocatoria de proyectos de Investigación Regionalización 2017.

2. IPS Universitaria de la Universidad de Antioquia, Clínica León XIII, por permitirnos desarrollar el piloto de este estudio.

\section{Conflicto de intereses}

Proyecto financiado a través del Comité para el Desarrollo de la Investigación de la Universidad de Antioquia, convocatoria de proyectos de Investigación, Regionalización 2017.

\section{Contribución de los autores}

TMVZ Contribuyó con la concepción y diseño del trabajo; adquisición, análisis e interpretación de los datos; y revisó de forma sustancial el artículo final.

DCMP Contribuyó con la concepción y diseño del trabajo; adquisición, análisis e interpretación de los datos; y revisó de forma sustancial el artículo final.

JFGC Contribuyó con la adquisición, análisis e interpretación de los datos; y revisó de forma sustancial el artículo final.

PAEV Contribuyó con la concepción y diseño del trabajo; y revisó de forma sustancial el artículo final.

CEVB Contribuyó con la concepción del trabajo; adquisición, análisis e interpretación de los datos; y revisó de forma sustancial el artículo final.

\section{Referencias}

1. Farrohknia N, Castrén M, Ehrenberg A, Lind L, Oredsson S, Jonsson H, Et al. Emergency department triage scales and their components: a systematic review of the scientific evidence. Scand J Trauma Resusc Emerg Med. 2011 Jun 30;19:42. https://doi.org/10.1186/1757-7241-19-42

2. World Health Organization. Emergency Triage Assessment and Treatment (ETAT): Manual for Participants. 2nd ed. Geneve: WHO; 2005. p. 78.

3. Oredsson S. Triage and patient safety in emergency departments. BMJ. 2011 Oct 20;343:d6652. https://doi. org/10.1136/bmj.d6652

4. Christ M, Grossmann F, Winter D, Bingisser R, Platz E. Modern triage in the emergency department. Dtsch Arztebl Int. 2010 Dec;107(50):892-8. https://doi.org/10.3238/arztebl.2010.0892

5. FitzGerald G, Jelinek GA, Scott D, Gerdtz MF. Emergency department triage revisited. Emerg Med J. 2010 Feb;27(2):86-92. https://doi.org/10.1136/emj.2009.077081

6. Wuerz R. Emergency severity index triage category is associated with six-month survival. ESI Triage Study Group. Acad Emerg Med. 2001 Jan;8(1):61-4. https://doi.org/10.1111/j.1553-2712.2001.tb00554.x

7. Platts-Mills TF, Travers D, Biese K, McCall B, Kizer S, LaMantia M, Et al. Accuracy of the Emergency Severity Index triage instrument for identifying elder emergency department patients receiving an immediate life-saving intervention. Acad Emerg Med. 2010 Mar;17(3):238-43. https://doi.org/10.1111/j.15532712.2010.00670.x 
8. Zachariasse JM, Seiger N, Rood PP, Alves CF, Freitas P, Smit FJ, Roukema GR, Et al. Validity of the Manchester Triage System in emergency care: A prospective observational study. PLoS One. 2017 Feb 2;12(2):e0170811. https://doi.org/10.1371/journal.pone.0170811

9. Lee JY, Oh SH, Peck EH, Lee JM, Park KN, Kim SH, Et al. The validity of the Canadian Triage and Acuity Scale in predicting resource utilization and the need for immediate life-saving interventions in elderly emergency department patients. Scand J Trauma Resusc Emerg Med. 2011 Nov 3;19:68. https://doi.org/10.1186/17577241-19-68

10. Colombia. Ministerio de salud y protección social. Resolución 5596 del 2015. Bogotá: El Ministerio; 2015.

11. Colombia. Ministerio de Salud y Protección Social. Modelo Integral de Atención en Salud - MIAS Plan de capacitación sectorial. MIAS. 2016.

12. Onder G, Rezza G, Brusaferro S. Case-Fatality Rate and Characteristics of Patients Dying in Relation to COVID-19 in Italy. JAMA. 2020 May 12;323(18):1775-6. https://doi.org/10.1001/jama.2020.4683

13. Rodríguez-Páez F, Jiménez-Barbosa R, Palencia-Sánchez F. Uso de los servicios de urgencias en Bogotá, CoIombia: Un análisis desde el Triaje Introducción. Univ y Salud. 2018;20(3):215-26. https://doi.org/10.22267/ rus.182003.124

14. Vargas. GN, González. MF, Menéndez. SE, Villaveces. M. Comparación de parametros de atención en una escala de triage de 5 niveles con los parametros estandar [internet]. Universidad Colegio Mayor de Nuestra Señora del Rosario; 2015. Disponible en: https://repository.urosario.edu.co/bitstream/handle/10336/10653/52709924-2015.pdf? sequence=1

15. Antioquia D S de S de. Indicadores Básicos de Salud Consolidado por Subregión y Municipio año 2016 [internet]. Antioquia, Dirección Seccional de Salud. Medellín; 2016. Disponible en: https://www.dssa.gov. co/index.php/estadisticas/mortalidad

16. Organization WH, Naciones Unidas P para el D. Antioquia, Retos y prioridades del Departamento 2016 2019 [Internet]. Programa de Naciones Unidas para el Desarrollo. Medellín; 2016. Disponible en: https:// www.co.undp.org/content/colombia/es/home/library/democratic_governance/retos-y-prioridades-deldepartamento-2016---20190.html

17. Cómo Vamos M. Informe de Calidad de Vida de Medellín 2014 [internet]. Medellín; 2014. Available from: http://www.medellincomovamos.org/salud

18. Ider E, Johnston AN, Crilly J. Review article: systematic review of three key strategies designed to improve patient flow through the emergency department. Emerg Med Australas. 2015 Oct;27(5):394-404. https:// doi.org/10.1111/1742-6723.12446

19. Chatterjee P, Cucchiara BL, Lazarciuc N, Shofer FS, Pines JM. Emergency department crowding and time to care in patients with acute stroke. Stroke. 2011 Apr;42(4):1074-80. https://doi.org/10.1161/STROKEAHA.110.586610

20. Affleck A, Parks P, Drummond A, Rowe BH, Ovens HJ. Emergency department overcrowding and access block. CJEM. 2013 Nov;15(6):359-84. English, French. https://doi.org/10.1017/s1481803500002451

21. Schull MJ, Slaughter PM, Redelmeier DA. Urban emergency department overcrowding: defining the problem and eliminating misconceptions. CJEM. 2002 Mar;4(2):76-83. https://doi.org/10.1017/ s1481803500006163

22. Oredsson S, Jonsson H, Rognes J, Lind L, Göransson KE, Ehrenberg A, Et al. A systematic review of triagerelated interventions to improve patient flow in emergency departments. Scand J Trauma Resusc Emerg Med. 2011 Jul 19;19:43. https://doi.org/10.1186/1757-7241-19-43

23. Robinson DJ. An integrative review: triage protocols and the effect on ED length of stay. J Emerg Nurs. 2013 Jul;39(4):398-408. https://doi.org/10.1016/j.jen.2011.12.016

24. Rowe BH, Villa-Roel C, Guo X, Bullard MJ, Ospina M, Vandermeer B, Et al. The role of triage nurse ordering on mitigating overcrowding in emergency departments: a systematic review. Acad Emerg Med. 2011 Dec;18(12):1349-57. https://doi.org/10.1111/j.1553-2712.2011.01081.x 
25. Moll HA. Challenges in the validation of triage systems at emergency departments. J Clin Epidemiol. 2010 Apr;63(4):384-8. https://doi.org/10.1016/j.jclinepi.2009.07.009

26. Kumar A, Lakshminarayanan D, Joshi N, Vaid S, Bhoi S, Deorari A. Triaging the triage: reducing waiting time to triage in the emergency department at a tertiary care hospital in New Delhi, India. Emerg Med J. 2019 Sep;36(9):558-563. https://doi.org/10.1136/emermed-2019-208577

27. Yuzeng S, Hui LL. Improving the wait time to triage at the emergency department. BMJ Open Qual. 2020 Feb;9(1):e000708. https://doi.org/10.1136/bmjoq-2019-000708

28. Fonarow GC, Zhao X, Smith EE, Saver JL, Reeves MJ, Bhatt DL, Et al. Door-to-needle times for tissue plasminogen activator administration and clinical outcomes in acute ischemic stroke before and after a quality improvement initiative. JAMA. 2014 Apr 23-30;311(16):1632-40. https://doi.org/10.1001/jama.2014.3203

29. García-Gigorro R, Cruz F De, Andrés-Esteban EM, Chacón-Alves S. Impact on patient outcome of emergency department length of stay prior to ICU admission. Med Intensiva. 2017;41(4):201-8. https://doi. org/10.1016/j.medine.2016.05.004

30. Vallejo CE, Patiño-Lugo DF, Aguirre-Acevedo DC. Effect of the Colombian Emergency Department Triage Policy on the timeliness of ED care and the mortality in patients with cerebrovascular attack : A controlled interrupted time series análisis. Health Serv Res. 2020;1(18). https://doi.org/10.21203/rs.3.rs-55373/v1

31. Ming T, Lai A, Lau PM. Can Team Triage Improve Patient Flow in the Emergency Department? A Systematic Review and Meta-Analysis. Adv Emerg Nurs J. 2016 Jul-Sep;38(3):233-50. https://doi.org/10.1097/ TME.0000000000000113

32. Hardy A, Calleja P. Triage education in rural remote settings: A scoping review. Int Emerg Nurs. 2019 Mar;43:119-125. https://doi.org/10.1016/j.ienj.2018.09.001

33. Subbe CP, Bottle RA, Bell D. Acute Medicine: triage, timing and teaching in the context of medical emergency admissions. Eur J Intern Med. 2011 Aug;22(4):339-43. https://doi.org/10.1016/j.ejim.2011.05.015

34. Madsen M, Kiuru S, Castrèn M, Kurland L. The level of evidence for emergency department performance indicators: systematic review. Eur J Emerg Med. 2015 Oct;22(5):298-305. https://doi.org/10.1097/ MEJ.0000000000000279

35. Geiderman JM, Malik S, McCarthy JJ, Jagoda A. The care of VIPS in the emergency department: Triage, treatment and ethics. Am J Emerg Med. 2018 Oct;36(10):1881-1885. https://doi.org/10.1016/j. ajem.2018.07.009 